\title{
城市热岛效应对天津市居住建筑 供暖和制冷负荷的影响
}

\author{
孟凡超 ${ }^{1}$, 任国玉 ${ }^{2,3^{*}}$, 郭 军 ${ }^{1}$, 张 雷 ${ }^{4}$, 张瑞雪 $^{5}$ \\ (1. 天津市气候中心, 天津 $300074 ; 2$. 中国气象局气候研究开放实验室/国家气候中心, 北京 100081 ; \\ 3. 中国地质大学(武汉)环境学院大气科学系, 武汉 430074; 4. 中国气象局国家气象信息中心, 北京 100081; \\ 5. 中国建筑科学研究院有限公司, 北京 100013)
}

\begin{abstract}
摘 要: 城市热岛效应已对建筑能源需求产生了重要影响,评估城市热岛效应影响下建筑的真实能耗需求及城乡差 异对既有建筑的节能调控和未来建筑的方案设计都具有重要意义。论文以天津自动气象站 2009-2017年逐时观 测数据为基础, 应用卫星遥感选站方法, 选取天津市区周围 4 个有代表性的乡村参考气象站, 对典型居住建筑全年 逐时负荷进行了动态模拟, 定量评估了城市热岛强度对不同时间尺度(年、日和小时)建筑负荷的影响。结果表明: (1) 随着城市热岛强度 $\left(I_{\mathrm{UHI}}\right)$ 的增强, 城市居住建筑供暖负荷减少、制冷负荷增加, 且年平均供暖负荷的减小幅度大 于年平均制冷负荷的增加幅度。 $I_{\mathrm{UHI}}$ 每上升 $1{ }^{\circ} \mathrm{C}$, 城市年平均供暖负荷较乡村减少 $4.01 \mathrm{kWh} / \mathrm{m}^{2}$ 、年平均制冷负荷增 加 $1.05 \mathrm{kWh} / \mathrm{m}^{2}$ 。(2) 冬季供暖期和夏季制冷期逐日负荷变化表现为:供暖期的高负荷时段主要集中在 12 月下旬至 翌年 1 月下旬、制冷期为 7 月下旬至 8 月上旬, 高负荷时段城市日平均供暖和制冷负荷分别较乡村约减少 $10 \%$ 、增 加 6\%。(3) 日内供暖负荷和制冷负荷小时变化均表现为夜间强于白天。在供暖期和制冷期, 北京时间 18:00 至次 日 07:00 时段无论在城市或是乡村都是高负荷时段; 11:00 至 15:00 时段在供暖期是低负荷时段,而在制冷期是高负 荷时段, 这可能与气温和供暖制冷需求有关。研究表明, 应充分考虑小时、日尺度热岛强度对用能的影响, 提高供 暖和空调运行调控的精细化水平, 以期达到降低供暖和制冷能耗的目的。
\end{abstract}

关 键 词: 城市热岛效应; 乡村参考站; 供暖和制冷负荷; 天津

中国建筑能耗占社会终端能源总消耗的 $1 / 3$ 左 右, 与工业能耗、交通能耗并列为中国 3 大用能领 域,而且该比例将随着城市化水平及人们对居住环 境需求的提高而进一步增加 ${ }^{[1-2]}$ 。建筑节能减排不 仅是缓解中国能源供需矛盾的关键所在, 也是确保 2030 年碳排放达标的关键之一 ${ }^{[3]}$ 。在建筑总能耗 中, 供暖和制冷能耗占据主导, 且与外界气候条件 有直接的关系 ${ }^{[4]}$ 。城市热岛 (Urban Heat Island, $\mathrm{UHI}$ )效应是城市气候最显著的特征之一 ${ }^{[5-6]}$, 已成 为 21 世纪面临的重要生态环境问题。建筑能耗受
城市热岛效应影响显著 ${ }^{[-9]}$, 研究城市热岛效应对建 筑供暖和制冷能耗的影响对降低社会终端总能耗、 减少污染气体排放及推进中国生态文明建设具有 重要意义。

大量研究表明,城市热岛效应对建筑能耗有着 极为重要的影响 ${ }^{[10-11]}$ 。 $\mathrm{Li}$ 等 ${ }^{[2]}$ 综述了近年来各国学 者应用不同方法研究的全球代表城市热岛效应对 建筑能耗的影响,认为城市热岛效应使供暖需求下 降、制冷需求上升,供暖能耗平均减少 $18.7 \%$ 、制冷 能耗平均增加 $19.0 \%$ 。城市热岛效应和全球变暖对

收稿日期:2019-09-18; 修订日期:2019-11-18。

基金项目: 国家重点研发计划项目(2018YFA0606302,2018YFA0605603); 天津市气象局博士基金项目(201744bsjj04)。[Foundation: National Key Research and Development Program of China, No. 2018 YFA0606302 and 2018YFA0605603; Doctoral Fund of Tianjin Meteorological Service, No. 201744bsjj04. ]

第一作者简介:孟凡超(1983-), 女, 博士, 工程师, 主要从事城市气候与建筑节能研究。E-mail: mfc08014072@163.com *通信作者简介: 任国玉(1958- ), 男,博士, 研究员, 主要从事气候变化研究。E-mail: guoyoo@cma.gov.cn in Tianjin City, China. Progress in Geography, 2020, 39(8): 1296-1307. ] DOI: 10.18306/dlkxjz.2020.08.005 
建筑用电需求和耗电量影响的综述总结了 11 个相 关研究得到: 温度每增加 $1{ }^{\circ} \mathrm{C}$, 峰值电负荷增加 $0.45 \% \sim 4.6 \%{ }^{[12]}$ 。针对城市热岛效应对不同气候区 建筑能耗影响的研究指出, 当忽略热岛效应时, 在 以供暖为主的冷气候区的建筑用能总量被明显高 估了, 在以制冷为主的热气候区的建筑用能总量则 被低估了,而对温和气候区影响不大 ${ }^{[13]}$ 。热岛效应 对建筑能耗的影响在不同时间尺度上表现不同。 在季节尺度上, 研究认为城市热岛效应在冬季较夏 季更加明显 ${ }^{[14]}$, 使冬季供暖能耗降低 ${ }^{[15]}$, 针对居住建 筑的研究认为夏季制冷能耗的增幅小于冬季供暖 能耗的降幅 ${ }^{[15]}$ 。在昼夜尺度上, 研究发现, 城市热 岛效应在夜间强于白天, 导致城区热度日数减小、 冷度日数增大 ${ }^{[16]}$ 。

近年来, 中国学者就城市热岛效应对建筑能耗 的影响开展了一些有意义的研究。建筑物暖通空 调系统消耗的能量大小取决于建筑物的供暖和制 冷负荷, 它包括围护结构的负荷、室内负荷和新风 负荷, 而城市热岛效应引起的气温升高对围护结构 负荷和新风负荷都有较大影响 ${ }^{[17]}$ 。夏季室内设定 温度越低, 热岛效应对建筑制冷能耗影响越大, 而 冬季恰好相反 ${ }^{[17]}$ 。气温升高对中国不同气候区建 筑能耗影响显著 ${ }^{[18]}$ 。在北方供暖区, 气温的升高使 供暖需求呈现减小趋势 ${ }^{[18]}$, 而制冷需求呈现增加趋 势 ${ }^{[19-20]}$; 陈莉等 ${ }^{[21]}$ 指出, 理论上由气温升高导致的中 国北方地区供暖耗能降低了 5\% 30\%, 长江中下游 地区供暖耗能降低了 $30 \%$ 以上。对过去和未来的 气温变化对能耗需求影响的研究表明,过去 $50 \mathrm{a}$, 华东供暖度日减少而降温度日增加, 供暖度日的减 少远超过降温度日的增加, 气温变化总体上有利于 建筑耗能的减少; 未来 $30 \mathrm{a}$, 华东供暖和降温度日 的变化幅度接近, 气温变化对建筑耗能的正负影响 将基本相抵消 ${ }^{[22]}$ 。

从上述研究现状可以看出, 国内外都在城市热 岛效应对能源需求及能耗影响的研究方面取得了 很多成果。但是, 基于多年实测小时气象数据对城 市热岛影响下建筑负荷在精细时间尺度(如逐时、 逐日)上的变化规律仍不清楚, 使精细化供暖制冷 调控缺乏科学依据。基于统计分析法(如度日法等) 的研究, 缺少对日气温的波动, 以及湿度、风速等多 气候要素的考虑, 而且没有考虑建筑本身的特点, 难以准确评估城市热岛效应对建筑用能的影响。 能耗模拟方法既考虑了多气象要素动态变化, 又结
合了建筑本身的特点, 是研究城市气候对建筑能耗 动态影响的主要方法。长期以来, 由于缺少多年实 测小时气温资料, 前期研究多使用其他替代方法或 者插值方法获取逐时气象数据 ${ }^{[23-24]}$, 而采用多年实 测逐时数据进行能耗模拟进而开展的相关研究相 对较少。另外,在城市热岛效应对能源影响的相关 研究中, 乡村参考气象站的选取至关重要。已有研 究中极少有应用气候学方法选取乡村参考气象站 进而模拟城市热岛效应对建筑能耗影响的工作, 有 必要采用更客观、科学的方法选取乡村参考站, 更 加准确地剥离出城市热岛效应对建筑能耗的影 响。因此,本文利用天津市自动站实测逐时气温资 料, 通过相对客观的标准选取乡村参考站, 以典型 居住建筑为研究对象, 应用瞬时系统模拟程序 (Transient System Simulation Program, TRNSYS) 软 件, 定量评估城市热岛强度变化对不同时间尺度 (年、日和小时)建筑供暖和制冷负荷的影响,可为政 府节能决策和供暖部门精细化供暖服务提供依据。

\section{1 数据与方法}

\section{1 研究区概况}

天津市 $\left(38^{\circ} 34^{\prime} \sim 40^{\circ} 15^{\prime} \mathrm{N} 、 116^{\circ} 43^{\prime} \sim 118^{\circ} 04^{\prime} \mathrm{E}\right)$ 位 于华北平原东部, 西有太行、北依燕山、东临渤海, 总面积约为 $11919.7 \mathrm{~km}^{2}$, 地处北温带半干旱半湿润 季风气候区, 四季分明。适宜的气候、丰富的河流， 以至河运、海运发达; 宽阔的平原, 陆路交通也十分 便利。这些使天津发展为中国北方最重要的工商 业繁荣的经济中心，也是中国北方最大的国际港口 城市。自改革开放以来, 天津经历了快速的城市化 进程, 城市人口不断增长, 城市区域迅速扩张, 下垫 面性质发生剧烈变化, 人为热排放增多, 环境污染 等因素导致城市热岛效应逐步加强。其中,市内六 区(和平区、河西区、河北区、河东区、南开区和红桥 区)是天津市工商业活动密集区,也是城市化进程 较快区(图 1), 近年来城市建设向新四区(西青区、东 丽区、津南区和北辰区)扩张。

\section{2 研究方法}

\subsection{1 乡村参考站的选取}

首先, 根据 Ren 等 ${ }^{[25]}$ 和 Yang 等 ${ }^{[26]}$ 发展的卫星遥 感选站方法, 采用2016年 1 月 MODIS 卫星遥感反 演资料(MOD11A2)绘制陆地表面亮度温度等值线, 结合自动气象站在地表亮度温度场中的位置等信 


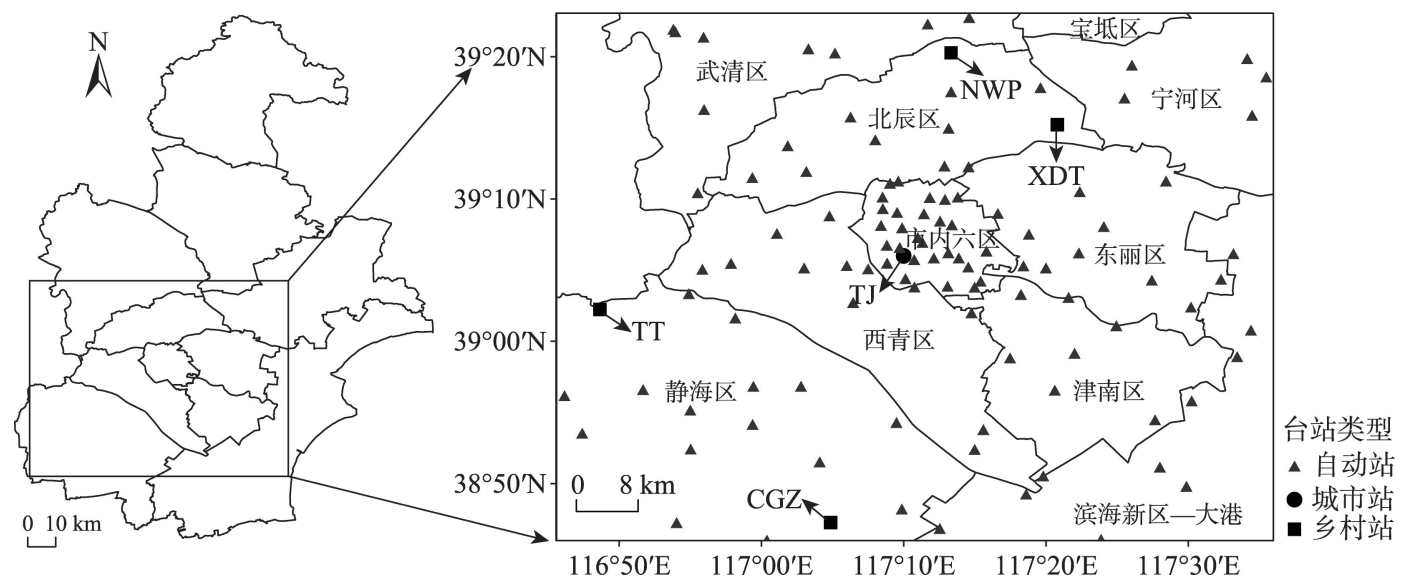

注: TJ 为天津城市站; TT、CGZ、NWP、XDT 分别为静海台头、静海蔡公庄、北辰南王平和北辰西堤头乡村参考站。

图 1 研究区及城市站、乡村站和自动站示意图

Fig.1 Location of the study area and distribution of the urban weather station, rural weather stations, and auto-weather stations in the study area

息, 拟定静海台头 $(\mathrm{TT}) 、$ 静海蔡公庄 $(\mathrm{CGZ}) 、$ 北辰南 王平 (NWP)和北辰西堤头 (XDT) 4 个站为备选乡村 参考站(图 1)。与其他方法相比, 该方法不依赖社会 和经济等数据, 是一种相对客观的选站方法。然 后, 采用土地利用/覆盖类型数据, 以拟定参考站为 中心, 以 $4 \mathrm{~km}$ 为半径生成缓冲区数据, 计算该区域 城镇和建筑用地所占百分率。结果表明, TT、CGZ、 NWP 和 XDT 4 个站的缓冲区内城镇和建筑用地所 占百分率均在 $10 \%$ 以内, 可作为乡村参考站。土地 利用/覆盖类型数据来源于中国科学院资源环境科 学数据中心研发的 2015 年中国大陆土地利用/覆盖 类型数据集(http://www.resdc.cn)。最后, 结合台站 观测环境等信息最终确定市中心周围 TT、CGZ、 NWP 和 XDT4 个站作为乡村参考站。所选参考站 位于市中心的不同方位,均处于开阔的自然或人工 植被区域, 远离高大建筑物和其他人工设施的影 响。 4 个参考站平均海拔高度均为 $1 \mathrm{~m}$ 左右, 差异 很小, 无需进行高度订正, 能够确保准确地估算城 市热岛强度。同时, 本文选择位于天津市城市中心 的城市气候监测站为城市站。表 1 总结了所用气象
台站的基本信息。

\subsection{2 城市热岛强度的定义}

城市热岛强度指由于城市热岛效应等因素引 起的城乡之间气温的差异, 用 $I_{\mathrm{UHI}}$ 表示。城市热岛 强度的表达式如下：

$$
I_{\mathrm{UHI}}=T_{\mathrm{u}}-T_{\mathrm{r}}
$$

式中: $T_{\mathrm{u}}$ 为城市站的气温; $T_{\mathrm{r}}$ 为参考站的气温。

天津市供暖期为每年的 11 月 15 日至翌年 3 月 15 日 (天津市统一规定); 制冷期为 6-8 月, 此时为 天津夏季。

\subsection{3 建筑能耗模拟}

TRNSYS 由美国 Wisconsin-Madison大学 Solar Energy 实验室开发, 之后经过逐步改进升级, 目前 广泛应用版本为 TRNSYS (v17.0)。TRNSYS 是模 块化的软件, 将整个暖通空调系统中的各部件模块 化, 按照各部件的数学模型, 以能质平衡为理论, 计算各出人口的温度、流量和压力等 ${ }^{[27]}$ 。主要模 块包括: 调用模块(Simulation Studio)、建筑物模型 (TRNBuild)及末端程序(TRNEdit)等,调用模块可用 于搭建暖通空调系统模型、制定控制策略等, 是

表 1 所用气象台站信息

Tab.1 Information of the weather stations used in this study

\begin{tabular}{clllccc}
\hline 台站类型 & 站号 & \multicolumn{1}{c}{ 站名 } & 缩写 & 经度 $/\left({ }^{\circ} \mathrm{E}\right)$ & 纬度 $/\left({ }^{\circ} \mathrm{N}\right)$ & 海拔 $/ \mathrm{m}$ \\
\hline 城市站 & 54517 & 天津 & TJ & 117.20 & 39.07 & 2.2 \\
乡村站 & $\mathrm{A} 2565$ & 静海台头 & TT & 116.81 & 39.04 & 1 \\
乡村站 & $\mathrm{A} 2566$ & 静海蔡公庄 & $\mathrm{CGZ}$ & 117.08 & 38.79 & 1 \\
乡村站 & $\mathrm{A} 3405$ & 北辰南王平 & NWP & 117.22 & 39.34 & 1 \\
乡村站 & $\mathrm{A} 3465$ & 北辰西堤头 & XDT & 117.35 & 39.25 & 1 \\
\hline
\end{tabular}


TRNSYS 软件的核心程序 ${ }^{[28]}$ 。特定的模块具有特定 的功能,可通过各模块组合、连接构成研究所需的 系统 ${ }^{[2]}$ 。TRNSYS 模拟结果具有较高的精准度, 广 泛应用于建筑和能源领域的模拟研究中, 是经建筑 行业普遍认可的商业化软件 ${ }^{[29-30]}$ 。

在中国,应用该软件对哈尔滨、天津、上海、广 州等城市的建筑能耗模拟研究均表明该系统具有 较好的模拟能力 ${ }^{[18,31-32]}$ 。应用 TRNSYS 对天津建筑 能耗进行模拟并监测能耗实际动态变化, 结果表 明: 该系统模拟小时能耗与实测小时能耗数据吻合 度较高, 误差能达到 $15 \%$ 以内, 认为该软件模拟结 果可反映真实能耗变化, 在研究气候对能耗的影响 方面是可靠的 ${ }^{[33]}$ 。

为完成能耗的模拟, 需要输人 2 类初始数据。 一类是 2009-2017年逐时气象数据, 包括太阳辐射 法向直射辐照度、水平面太阳总辐射、气温、相对湿 度、风速、风向等数据, 均来自于天津市气象局气象 信息中心, 经过严格质控 ${ }^{[34]}$ 。其中, 太阳辐射法向 直射辐照度逐时数据参照《建筑节能气象参数标 准》(JGJT346-2014)中水平面太阳总辐射的直散分 离模型计算获得。另一类是与能耗相关的建筑参 数, 主要包括建筑围护结构传热系数、制冷或供暖 期建筑物室内设计条件、室内得热参数和窗墙比 等, 参照《严寒和寒冷地区居住建筑节能设计标准》 (JGJ26-2010)选取(表2)。

\section{2 结果分析}

\section{1 全年气候因子变化特征}

\subsection{1 $I_{\mathrm{UHI}}$ 逐日变化}

2009－2017年全年城市和乡村气温及二者差 值(城市热岛强度 $I_{\mathrm{UHI}}$ )逐日变化如图 2 所示。总体来 看, 城市和乡村气温的逐日变化趋势基本一致,二 者差异秋季和冬季较大、春季和夏季较小, 全年均 出现城市热岛现象。9 a 来, 供暖期(11月 15 日至翌 年 3 月 15 日)平均 $I_{\mathrm{UHI}}$ 为 $2.0{ }^{\circ} \mathrm{C}, 12$ 月末至 1 月末 $I_{\mathrm{UHI}}$
最强, 最高达到 $3.0^{\circ} \mathrm{C}(1$ 月 4 日和 9 日); 制冷期 $(6$ 月 1 日一 8 月 31 日)平均 $I_{\mathrm{UHI}}$ 为 $1.1{ }^{\circ} \mathrm{C}, 8$ 月中下旬 $I_{\mathrm{UHI}}$ 最 强, 最高达到 $1.7^{\circ} \mathrm{C}(8$ 月 25 日 $)$ 。

\subsection{2 相对湿度逐日变化}

全年城乡相对湿度及其差值逐日变化(图 3)显 示, 城市全年相对湿度逐日变化均小于乡村, 在第 200 天(7月中旬)之后, 城乡差距明显变大。冬季供 暖期, 城乡相对湿度变化不大, 城市相对湿度为 $30.0 \% \sim 60.5 \%$, 乡村为 $36.0 \% \sim 70.5 \%$, 城乡相对湿度 差平均值为 $10.2 \%$ 。夏季制冷期(6-9 月), 城乡日 相对湿度均呈现为先上升后下降的趋势, 城市相对 湿度为 $43.7 \% \sim 73.7 \%$, 乡村为 $51.0 \% \sim 85.0 \%$, 城乡相 对湿度差平均值为 $10.0 \%$ 。

\subsection{3 风速逐日变化}

图 4 所示为城乡风速及其差值全年逐日变化, 城市风速全年逐日值均小于乡村, 城市风速变化波 动不大, 而乡村风速春季最大、夏季最小。冬季供 暖期城市日平均风速为 $1.42 \mathrm{~m} / \mathrm{s}$, 乡村为 $2.36 \mathrm{~m} / \mathrm{s}$, 城乡风速差较大。夏季制冷期城乡风速差逐渐变 小, 城市日平均风速为 $1.44 \mathrm{~m} / \mathrm{s}$, 乡村为 $1.97 \mathrm{~m} / \mathrm{s}$, 相 差较小。

\subsection{4 建筑负荷对气候因子的响应}

李红莲等 ${ }^{[35}$ 指出: 建筑的负荷是指为达到期望 状态需要补充或去除热量的大小。本文分析供暖 期和制冷期居住建筑负荷差值 $(y)$ 与城乡气温差 $\left(I_{\mathrm{UHI}}\right)$ 、相对湿度差 $(\triangle \mathrm{RH})$ 和风速差的相关性。在供 暖期, 只有 $I_{\mathrm{UHI}}$ 进入模型: $y=-37.054 \times I_{\mathrm{UHI}}+10.733$, 决 定系数 $R^{2}=0.829(n=968, P<0.001)$, 说明 $I_{\mathrm{UHI}}$ 对城乡 供暖期负荷差起主导作用, $I_{\mathrm{UHI}}$ 可以解释负荷差变化 的 $82.9 \%$ 。在制冷期, $I_{\mathrm{UHI}}$ 和相对湿度差 2 个因子进 人了模型, 其中 : 包含 $I_{\mathrm{UHI}}$ 的模型为 $y=9.139 \times I_{\mathrm{UHI}}+$ 26.383, $R^{2}=0.117(n=828, P<0.001)$, 而包含 $I_{\mathrm{UHI}}$ 和相 对湿度差 2 个因子的模型为 $y=29.546 \times I_{\mathrm{UHI}}+1.817 \times \triangle$ $\mathrm{RH}+20.894, R^{2}=0.440(n=828, P<0.001)$, 表明制冷负 荷除了受 $I_{\mathrm{UHI}}$ 外, 还受相对湿度差的影响。总之, 热 岛强度对供暖和制冷负荷具有明显影响。

表 2 中国寒冷地区居住建筑设计参数

Tab.2 Design parameters of residential buildings in the cold climate zone of China

\begin{tabular}{|c|c|c|c|c|c|c|c|c|c|c|c|c|}
\hline \multicolumn{3}{|c|}{ 建筑围护结构传热系数 $/\left(\mathrm{W} \cdot \mathrm{m}^{-2} \cdot{ }^{\circ} \mathrm{C}^{-1}\right)$} & \multicolumn{3}{|c|}{ 室内设计条件夏季/冬季 } & \multicolumn{3}{|c|}{ 室内得热参数 } & \multicolumn{4}{|c|}{ 窗墙比 } \\
\hline 墙 & 屋顶 & 窗户 & 温度 $/{ }^{\circ} \mathrm{C}$ & $\begin{array}{c}\text { 相对湿度 } \\
1 \%\end{array}$ & $\begin{array}{c}\text { 新风量/ } \\
\left(\mathrm{m}^{3} / \mathrm{h}\right)\end{array}$ & $\begin{array}{c}\text { 人员密度/ } \\
\left(\mathrm{m}^{2} / \text { 人) }\right.\end{array}$ & $\begin{array}{c}\text { 照明功率 } \\
\text { 密度 } /\left(\mathrm{W} / \mathrm{m}^{2}\right) \\
\end{array}$ & $\begin{array}{c}\text { 电器设备 } \\
\text { 功率/(W/m²) }\end{array}$ & 东 & 南 & 西 & 北 \\
\hline 0.60 & 0.45 & 2.50 & $26 / 18$ & $60 / 35$ & 30 & 32 & 5 & 5 & 0.28 & 0.41 & 0.28 & 0.41 \\
\hline
\end{tabular}



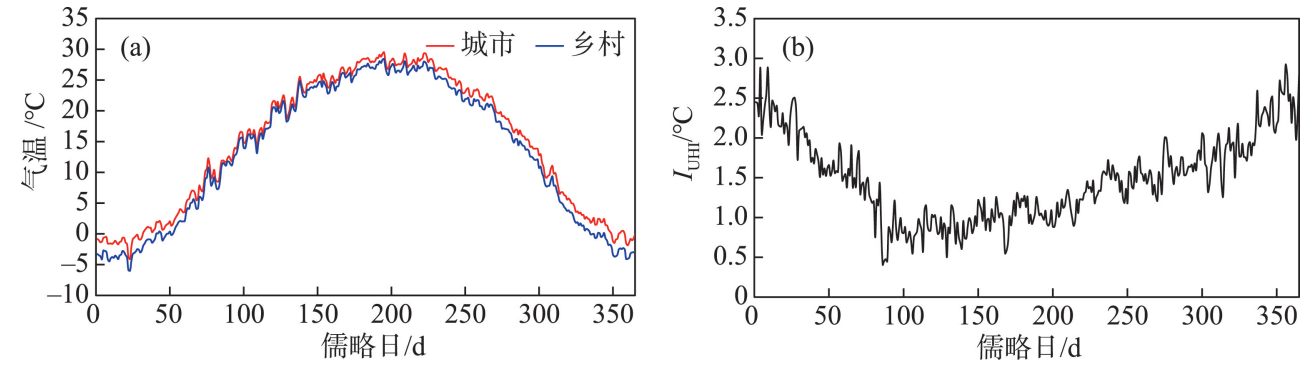

图 2 2009-2017年全年城市和乡村气温(a)及 $I_{\mathrm{UHI}}(\mathrm{b})$ 逐日变化

Fig.2 Daily variations of the temperature in urban and rural areas (a) and the $I_{\mathrm{UHI}}$ (b) during 2009-2017
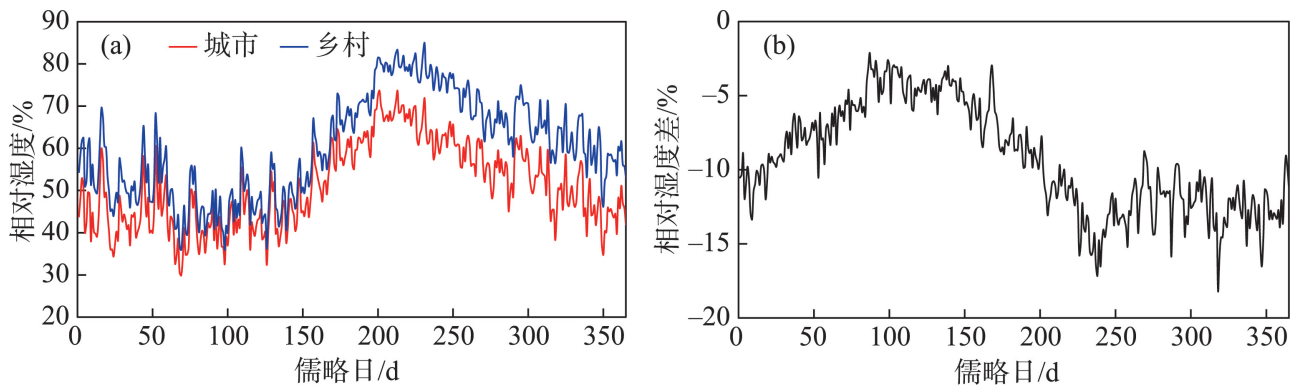

图 32009 - 2017年全年城市和乡村相对湿度(a)及其差值(b)逐日变化

Fig.3 Daily variations of the relative humidity in urban and rural areas (a), and their difference (b) during 2009-2017
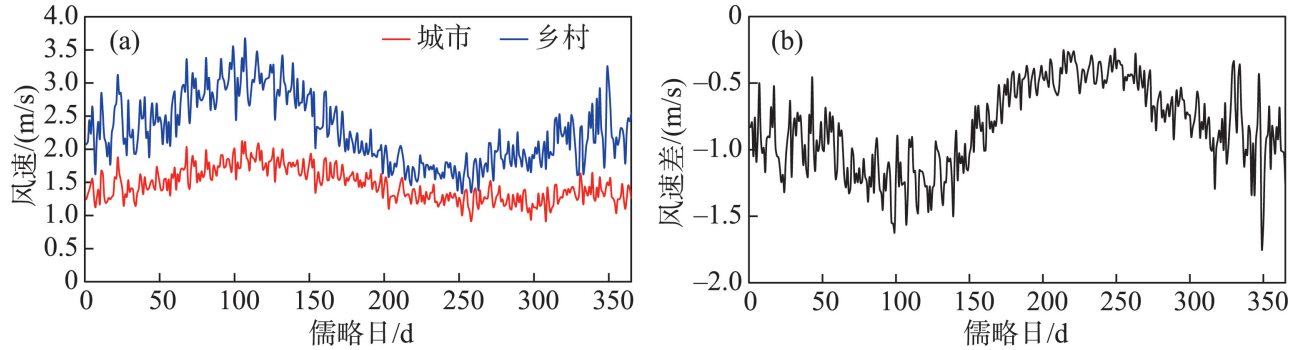

图４２009-2017年全年城市和乡村风速(a)及其差值(b)逐日变化

Fig.4 Daily variations of the wind speed in urban and rural areas (a) and their difference (b) during 2009-2017

\section{2 城市热岛强度对建筑供暖和制冷负荷的影响}

\subsection{1 冬季供暖期和夏季制冷期建筑负荷年变化}

供暖期,2009-2017年城市和乡村居住建筑负 荷均呈减少趋势, 城市供暖负荷较乡村平均每年减 少 $10.29 \%$ (图 5a)。制冷期,城市和乡村居住建筑负 荷呈不同程度的增加趋势, 城市供暖负荷较乡村平 均每年增加 7.48\%(图 5b)。从历年城乡负荷差值与 $I_{\mathrm{UHI}}$ 的相关分析来看, $I_{\mathrm{UHI}}$ 每上升 $1{ }^{\circ} \mathrm{C}$, 城市供暖负荷 较乡村减少 $4.01 \mathrm{kWh} / \mathrm{m}^{2}$ (图 5c), 而制冷负荷较乡村 增加 $1.05 \mathrm{kWh} / \mathrm{m}^{2}$ (图 5d)。

2.2.2 冬季供暖期和夏季制冷期建筑负荷逐日变化 2009-2017年城乡供暖期和制冷期平均负荷 逐日变化如图 6 和表 3 所示。从供暖期来看, 城市 和乡村供暖负荷变化趋势一致, 基本呈单峰分布, 城市始终低于乡村(图 6a)。由表3 可知, 城市高负
荷时段出现在 12 月 23 日至翌年 1 月 26 日，日平均 负荷值为 $0.6838 \mathrm{kWh} / \mathrm{m}^{2}$; 乡村高负荷时段出现在 12 月 24 日至翌年 1 月 26 日, 日平均负荷值达到 $0.7644 \mathrm{kWh} / \mathrm{m}^{2}$, 高负荷时段城市的日平均负荷值较 乡村减少 $10.54 \%$ 。城乡低负荷时段均出现在 3 月 11-15日，城市的日平均负荷值较乡村减少 $11.67 \%$ 。从逐日城乡供暖负荷差值与 $I_{\mathrm{UHI}}$ 的相关分 析来看, $I_{\mathrm{UHI}}$ 每上升 $1{ }^{\circ} \mathrm{C}$, 城市供暖负荷较乡村减少 $0.0330 \mathrm{kWh} / \mathrm{m}^{2}$ (图 6c)。

从夏季制冷期来看, 城市和乡村差别相对较 小,城市制冷负荷较高(图 6b)。制冷期高、低负荷时 段及其日平均负荷值如表 3 所示, 城乡高负荷时段 均出现在 7 月 27 日 -8 月 13 日,城市的日平均负荷 值较乡村增加 $6.27 \%$; 城市低负荷时段出现在 6 月 $1-11$ 日, 乡村出现在 6 月 $1-14$ 日,城市较乡村增 

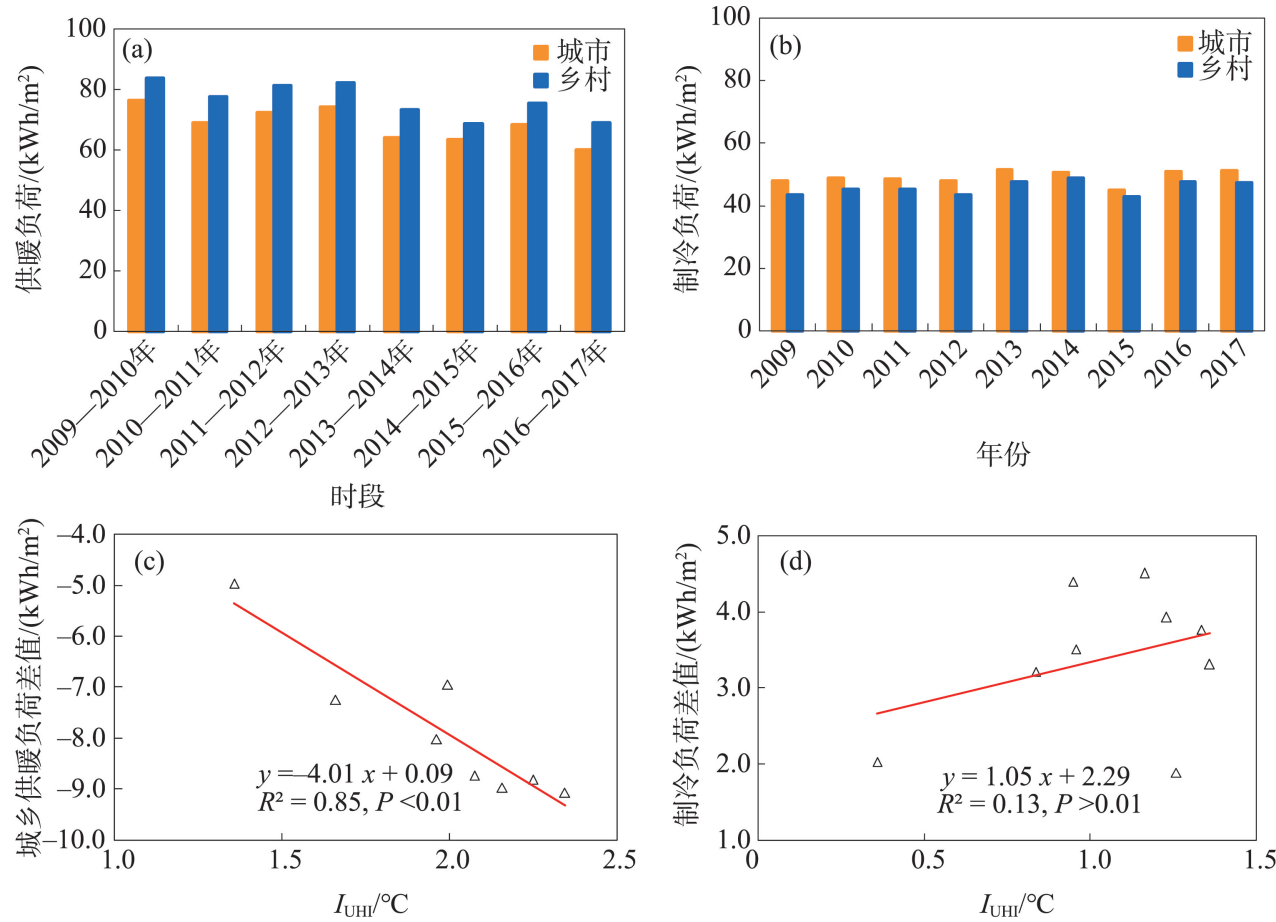

图 52009 - 2017年城市和乡村供暖和制冷年累积负荷变化(a、b)及其与 $I_{\mathrm{UHI}}$ 的相关性(c、d)

Fig.5 Annual variations of the heating/cooling loads in urban and rural areas $(a, b)$ and the correlation between the loads and $I_{\mathrm{UHI}}(\mathrm{c}, \mathrm{d}), 2009-2017$
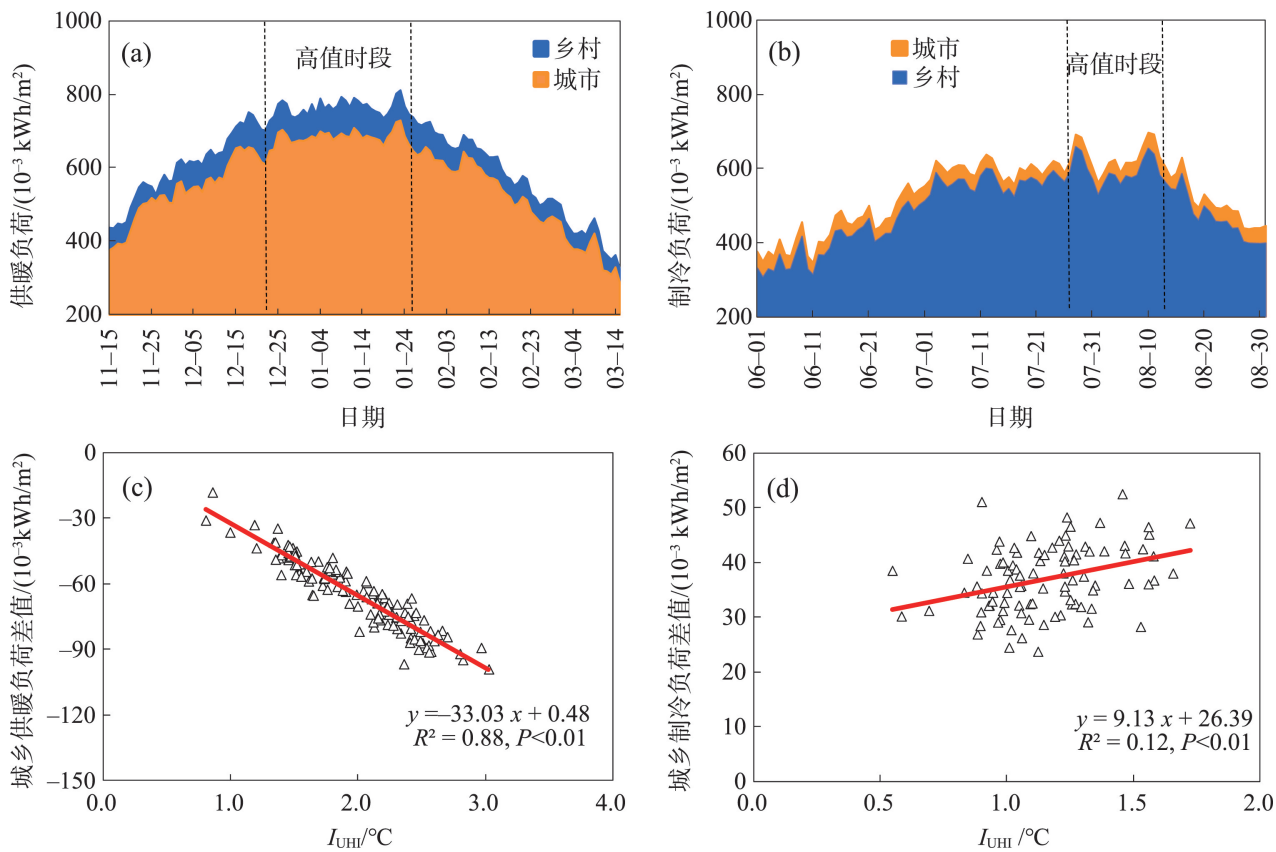

图 $62009-2017$ 年城市和乡村供暖制和冷负荷日变化 $(\mathrm{a} 、 \mathrm{~b})$ 及与 $I_{\mathrm{UHI}}$ 的相关性 $(\mathrm{c} 、 \mathrm{~d})$

Fig.6 Daily variations of the heating/cooling loads in urban and rural areas (a, b) and the correlation between the loads and $I_{\mathrm{UHI}}(\mathrm{c}, \mathrm{d}), 2009-2017$

加 $9.31 \%$ 。从逐日城乡制冷负荷差值与 $I_{\mathrm{UHI}}$ 的相关 分析来看, $I_{\mathrm{UHI}}$ 每上升 $1{ }^{\circ} \mathrm{C}$, 城市制冷负荷较乡村增 加 $0.0091 \mathrm{kWh} / \mathrm{m}^{2}$ (图 6d)。
2.2.3 冬季供暖期和夏季制冷期建筑负荷逐时变化 供暖期和制冷期年平均日内小时供暖和制冷 负荷分布如图 7和表4所示。在 2009-2017年供暖 
期间, 城市平均每小时负荷为 $0.0236 \mathrm{kWh} / \mathrm{m}^{2}$, 乡村 为 $0.0263 \mathrm{kWh} / \mathrm{m}^{2}$ (图 7a)。城乡供暖负荷日内均呈 现有规律的单峰分布, 二者趋势基本一致,均表现 为夜间强、白天弱,城市负荷始终小于乡村, 以夜间 尤甚。整体表现为 2 个平稳阶段和 2 个急剧变化时 段, 1 个平稳高值时段 18:00 至次日 07:00(北京时 间, 下同), 城市平均负荷值较乡村低 $11.63 \%$; 一个 平稳低值阶段 11:00 15:00, 城市平均负荷值较乡村 低 $5.83 \% ; 2$ 个急剧变化时段表现为, 16:00 18:00 为 快速上升时段,而 07:00 10:00 为快速下降时段。

在制冷期间, 城市平均每小时负荷为 0.0222 $\mathrm{kWh} / \mathrm{m}^{2}$, 乡村为 $0.0207 \mathrm{kWh} / \mathrm{m}^{2}$ (图 7b)。城乡制冷 负荷日内波动较大, 但城乡趋势仍然基本一致, 城
市高于乡村, 以夜间升高幅度更大。城市和乡村制 冷负荷高值时段有：(1) 18:00 至次日 07:00,城市制 冷负荷较乡村高 $9.84 \%$; 2 11:00 15:00, 城市较乡 村高 $3.68 \%$ 。这 2 个时段是人们休息的主要时段, 而 08:00 10:00 和 16:00 17:00 在一天中负荷最低, 这 2 个时段是人们上下班时间,负荷较低可能与此 有关。

\section{3 结论与讨论}

\section{1 结论}

本文应用经过质量控制的高密度自动气象站 逐时气温资料和客观遥感方法选择乡村参考站,

表3 2009-2017年供暖期和制冷期高、低负荷时段及其日平均负荷值

Tab.3 The high/low time period and the average daily loads in the heating/cooling period, 2009-2017

\begin{tabular}{|c|c|c|c|c|c|c|}
\hline \multirow{2}{*}{ 比较项目 } & \multicolumn{3}{|c|}{ 供暖期 } & \multicolumn{3}{|c|}{ 制冷期 } \\
\hline & 城市 & 乡村 & 城市较乡村变化 $/ \%$ & 城市 & 乡村 & 城市较乡村变化 $/ \%$ \\
\hline 高负荷时段 & 12月 23 日至 & 12月 24 日至 & & 7月 27 日一 & 7月 27 日一 & \\
\hline $\begin{array}{l}\text { 日平均高负荷值 } \\
/\left(10^{-3} \mathrm{kWh} / \mathrm{m}^{2}\right)\end{array}$ & $\begin{array}{l}\text { 翌年1月26日 } \\
683.8\end{array}$ & $\begin{array}{l}\text { 翌年1月26日 } \\
764.4\end{array}$ & -10.54 & $\begin{array}{l}8 \text { 月 } 13 \text { 日 } \\
629.9\end{array}$ & $\begin{array}{l}8 \text { 月 } 13 \text { 日 } \\
592.7\end{array}$ & 6.27 \\
\hline 低负荷时段 & 3 月 11 日一 -15 日 & 3 月 11 日一 -15 日 & & 6月 1 日一-11日 & 6 月 1 日一- 14 日 & \\
\hline $\begin{array}{l}\text { 日平均低负荷值 } \\
/\left(10^{-3} \mathrm{kWh} / \mathrm{m}^{2}\right)\end{array}$ & 313.3 & 354.7 & -11.67 & 379.2 & 346.9 & 9.31 \\
\hline
\end{tabular}
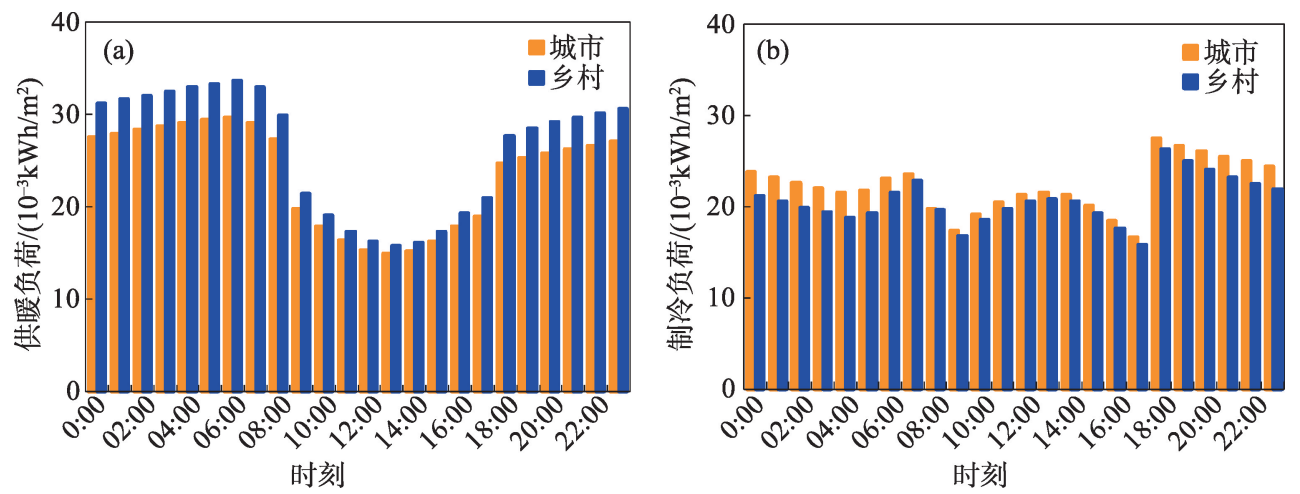

图 7 2009-2017年城市和乡村供暖(a)和制冷(b)负荷日内小时变化

Fig.7 Hourly variations of diurnal heating (a) / cooling (b) loads in the urban and rural areas, 2009-2017

表 $42009-2017$ 年供暖和制冷负荷平均日内变化平稳高、低值时段及其小时平均负荷值

Tab.4 The high/low time period of the diurnal cycle and the average hourly loads in the heating/cooling period, 2009-2017

\begin{tabular}{|c|c|c|c|c|c|c|}
\hline \multirow{2}{*}{ 比较项目 } & \multicolumn{3}{|c|}{ 供暖期 } & \multicolumn{3}{|c|}{ 制冷期 } \\
\hline & 城市 & 乡村 & 城市较乡村变化 $/ \%$ & 城市 & 乡村 & 城市较乡村变化 $/ \%$ \\
\hline 平稳高值时段 & $\begin{array}{l}\text { 18:00 } \\
\text { 次日 07:00 }\end{array}$ & $\begin{array}{l}\text { 18:00 } \\
\text { 次日 07:00 }\end{array}$ & & $\begin{array}{l}\text { 18:00 次日 } 07: 00, \\
11: 00 \sim 15: 00\end{array}$ & $\begin{array}{l}\text { 18:00 次日 } 07: 00, \\
11: 00 \sim 15: 00\end{array}$ & \\
\hline $\begin{array}{l}\text { 平均高负荷值/ } \\
\left(10^{-3} \mathrm{kWh} / \mathrm{m}^{2}\right)\end{array}$ & 27.54 & 31.17 & -11.63 & $24.06,20.96$ & $21.90,20.21$ & $9.84,3.68$ \\
\hline 平稳低值时段 & $11: 00 \sim 15: 00$ & $11: 00 \sim 15: 00$ & & - & - & \\
\hline $\begin{array}{l}\text { 平均低负荷值/ } \\
\left(10^{-3} \mathrm{kWh} / \mathrm{m}^{2}\right)\end{array}$ & 15.60 & 16.57 & -5.83 & - & - & - \\
\hline
\end{tabular}


对中国北方典型居住建筑供暖和制冷负荷进行了 逐时动态模拟计算, 分析了热岛强度对不同时间 尺度供暖和制冷负荷影响的趋势和大小, 得到以 下结论:

(1) 总体上, 城市较乡村热岛强度增加、相对湿 度和风速减小, 使居住建筑供暖负荷减少、制冷负 荷增加。城市年平均供暖负荷较乡村减少 $10.29 \%$ 、 年平均制冷负荷较乡村增加 $7.48 \%$ 。 $I_{\mathrm{UHI}}$ 是影响负 荷变化的主要气候因素。 $I_{\mathrm{UH}}$ 每上升 $1{ }^{\circ} \mathrm{C}$, 城市供暖 负荷较乡村减少 $4.01 \mathrm{kWh} / \mathrm{m}^{2}$ 、制冷负荷增加 1.05 $\mathrm{kWh} / \mathrm{m}^{2}$ 。

（2）居住建筑全年逐日负荷变化表现为: 随冬 季城市热岛强度的加强, 供暖期日平均负荷高于制 冷期日平均负荷。供暖期高负荷时段集中在 12 月 下旬至翌年1月下旬,制冷期高负荷时段出现在 7 月 下旬至 8 月上旬, 高负荷时段城市日平均供暖和制 冷负荷分别较乡村减少 $10 \%$ 左右和增加 $6 \%$ 左右。

(3) 居住建筑日内小时负荷影响在供暖期和制 冷期均表现为夜间强、白天弱。供暖负荷日内表现 为明显的平稳高值阶段(18:00 至次日 07:00)和低值 阶段(11:00 15:00), 城市平均负荷较乡村分别减少 $11.63 \%$ 和 $5.83 \%$; 制冷负荷日内变化规律性较弱, 制 冷高值包括 18:00 至次日 07:00 和 11:00 15:00 两个 阶段。总之, 18:00 到次日 07:00 不管在供暖期和制 冷期都是负荷需求较强时段; 11:00 15:00 在供暖期 是负荷低值时段, 而在制冷期是负荷高值时段, 原 因可能是该时段在供暖期和制冷期城乡气温均是 一天中的高值时段,供暖需求小, 而制冷需求大。

\section{2 讨论}

随着城市化进程的加快, 城市热岛效应不断增 强 ${ }^{[3]}$ 。城市热岛对建筑能耗产生了巨大影响 ${ }^{[37]}$, 尤其 是建筑的冷热能耗受热岛效应的影响极为明显 ${ }^{[12,38]}$ 。 城市热岛强度空间分布呈非均质性 ${ }^{[2,39]}$, 造成城市 和乡村的能耗需求有着一定的差别。对不同城市 热岛条件下城乡能耗需求差别了解更清晰, 可为科 学、精细化供暖和制冷提供依据。

实际上, 城市热岛发生时, 其他气象因素也在 发生变化, 它们共同影响着能源消耗 ${ }^{[30-31]}$ 。冬季采 暖能耗主要受气温的影响, 夏季制冷能耗受气温和 湿度的共同影响 ${ }^{[20,40]}$ 。本文研究得出, 城乡气温全 年城市大于乡村, 城市热岛强度秋冬高、春夏低, 冬 季最高达 $3.0^{\circ} \mathrm{C}$, 夏季最高为 $1.7^{\circ} \mathrm{C}$; 城市全年相对 湿度均小于乡村, 供暖期变化不大, 制冷期先上升
后下降;城市风速全年均小于乡村,乡村风速波动较 大。对建筑负荷与各气象因子的相关分析发现, 热 岛强度是居住建筑能耗需求的主要影响因子。

建筑的负荷是指为达到期望状态需要补充或 去除热量的大小 ${ }^{[35]}$ 。城市热岛对居住建筑年变化 影响上, 研究认为总体上城市热岛效应使建筑供暖 能耗下降、制冷能耗增加 ${ }^{[2,41]}$ 。本文研究结果表明, 供暖期城市负荷较乡村平均每年减少 $10.29 \%$, 制冷 期城市负荷较乡村平均每年增加 $7.48 \%$, 供暖负荷 的降幅高于制冷负荷的增幅, 与中国北方城市青岛 的变化趋势一致 ${ }^{[15]}$ 。此外, 本文研究还发现, 热岛 强度越大, 总制冷负荷越大, $I_{\mathrm{UHI}}$ 每上升 $1^{\circ} \mathrm{C}$, 城市供 暖负荷较乡村减少 $4.01 \mathrm{kWh} / \mathrm{m}^{2}$; $I_{\mathrm{UHI}}$ 每上升 $1^{\circ} \mathrm{C}$, 城 市制冷负荷较乡村增加 $1.05 \mathrm{kWh} / \mathrm{m}^{2}$ 。陈怡 ${ }^{[42]}$ 模拟 了 2015 年 7 月 3 日至 9 月 30 日广州居住建筑的负荷 动态变化, 得到热岛强度每增加 $1{ }^{\circ} \mathrm{C}$, 平均单位面 积总热负荷减少了 $1.22 \mathrm{kWh} / \mathrm{m}^{2}$, 总冷负荷平均增 加 $7.74 \mathrm{kWh} / \mathrm{m}^{2}$ 的结论。原因可能由于天津位于我 们北方寒冷气候区, 广州位于夏热冬暖气候区, 寒 冷地区冬季采暖需求相对较大, 而夏热冬暖气候区 夏季制冷时间更长、需求更大, 所以出现天津供暖 期城乡负荷差值更大、广州制冷期负荷差值更大的 差异现象。

本文研究发现, 热岛强度能够解释供暖期居住 建筑负荷变化的 $82.9 \%$, 是影响供暖负荷变化的主 要因素。在冬季供暖期, 城乡 $I_{\mathrm{UH}}$ 平均为 $2.0^{\circ} \mathrm{C}$, 最 高达 $3.0^{\circ} \mathrm{C}$, 且城市日平均风速较乡村小 $0.94 \mathrm{~m} / \mathrm{s}$ 。 $I_{\mathrm{UHI}}$ 每上升 $1{ }^{\circ} \mathrm{C}$, 供暖负荷差值升高 $0.0330 \mathrm{kWh} / \mathrm{m}^{2}$ 。 城乡供暖期负荷变化趋势一致,高负荷时段出现在 12 月下旬至 1 月下旬, 城市日平均负荷较乡村减少 10\%左右, 而供暖期开始和结束为低负荷时段。在 夏季制冷期,高负荷时段 (7月下旬至 8 月上旬)城市 日平均制冷负荷较乡村增加 6\%左右。制冷期城乡 $I_{\mathrm{UHI}}$ 平均为 $1.1^{\circ} \mathrm{C}$, 最高为 $1.7^{\circ} \mathrm{C}$, 较供暖期减弱。天 津市位于渤海西岸, 这种地理环境特征能使城市热 岛环流和海陆风环流相互耦合 ${ }^{[43]}$, 夏季海陆风效应 对城市热岛效应有一定的削弱作用 ${ }^{[8,44]}$, 从而使 $I_{\mathrm{UHI}}$ 有所减弱。 $I_{\mathrm{UHI}}$ 每上升 $1^{\circ} \mathrm{C}$, 城乡制冷负荷差值升高 $0.0091 \mathrm{kWh} / \mathrm{m}^{2}$ 。

城市热岛对居住建筑小时负荷变化影响方面, 本文研究得出,在 2009-2017年供暖期间,城市平

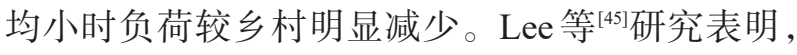
城市热岛效应使城市夜间最低气温升高, 这将有利 
于城市供暖节能。本文中城乡供暖负荷日内均表 现为夜间强、白天弱。全天 24 小时城乡负荷高值 时段从北京时间 18:00 至次日 07:00, 城市平均负荷 值较乡村低 $11.63 \%$, 此时供暖负荷受城市热岛效 应的影响较大。在小时尺度上, 刘玉莲等 ${ }^{[46]}$ 应用度 时法研究了哈尔滨采暖期内采暖强度随时间变化的 特征, 得出哈尔滨采暖期小时平均采暖强度 06:00 最大, 而 14:00 最小, 本文结果与之基本一致。 Tewari 等 ${ }^{[47}$ 通过分析美国南部不同地区日内小时 空调制冷用电能耗变化, 得到未来增加的制冷能耗 需求约有 $75 \%$ 是由于城市化影响、25\% 是由于全球 变暖影响的结论, 可见城市热岛对制冷负荷的影响 程度之大。本文研究发现, 城乡制冷负荷日内变化 规律不强, 但城乡趋势仍然基本一致, 城市始终高 于乡村,且夜间升高幅度更大。18:00 至次日 07:00 和 11:00 15:00 是城市和乡村制冷负荷的高值时 段,这 2 个时段是人们休息的主要时段, 居住建筑 使用空调的时间多集中在夜晚至次日早上 ${ }^{[4]}$, 而城 市热岛强度夜间强度大、白天小, 必然对相应的负 荷产生影响, 而夏季 11:00 15:00也正是一天中气 温的高值时段, 制冷负荷也随之增加。总之, 不管 供暖期还是制冷期, 夜间负荷需求均高于白天。本 文揭示了热岛效应影响下年、日、小时时间尺度居 住建筑供暖和制冷负荷的变化规律, 为相关部门在 精细化调控、尤其是高负荷时段供暖调控等方面提 供参考依据。

\section{参考文献(References)}

[1] 江亿. 我国建筑节能战略研究 [J]. 中国工程科学, 2011, 13(6): 30-38. [Jiang Yi. China building energy conservation stratagems study. Strategic Study of CAE, 2011, 13 (6): 30-38. ]

[2] Li X M, Zhou Y Y, Yu S, et al. Urban heat island impacts on building energy consumption: A review of approaches and findings [J]. Energy, 2019, 174: 407-419.

[3] 何正旭, 高浪. 我国城镇居住建筑能耗强度定量分析与 预测 [J]. 四川建筑, 2018, 38(3): 45-47. [He Zhengxu, Gao Lang. Quantitative analysis and prediction of energy consumption intensity of urban residential buildings in China. Sichuan Architecture, 2018, 38(3): 45-47. ]

[4] Huang J, Gurney K R. The variation of climate change impact on building energy consumption to building type and spatiotemporal scale [J]. Energy, 2016, 111: 137-153.

[5] Lowe S A. An energy and mortality impact assessment of the urban heat island in the US [J]. Environmental Impact
Assessment Review, 2016, 56: 139-144.

[6] 李元征, 尹科, 周宏轩, 等. 基于遥感监测的城市热岛研 究进展 [J]. 地理科学进展, 2016, 35(9): 1062-1074. [Li Yuanzheng, Yin Ke, Zhou Hongxuan, et al. Progress in urban heat island monitoring by remote sensing. Progress in Geography, 2016, 35 (9): 1062-1074. ]

[7] Sailor D J. Risks of summertime extreme thermal conditions in buildings as a result of climate change and exacerbation of urban heat islands [J]. Building and Environment, 2014, 78: 81-88.

[8] Palme M, Inostroza L, Villacreses G, et al. From urban climate to energy consumption. Enhancing building performance simulation by including the urban heat island effect [J]. Energy and Buildings, 2017, 145: 107-120.

[9] He B J. Towards the next generation of green building for urban heat island mitigation: Zero UHI impact building [J]. Sustainable Cities and Society, 2019, 101647. doi: 10.1016/j.scs.2019.101647.

[10] Gunawardena K, Kershaw T, Steemers K. Simulation pathway for estimating heat island influence on urban/ suburban building space-conditioning loads and response to facade material changes [J]. Building and Environment, 2019, 150: 195-205.

[11] Morakinyo T E, Ren C, Shi Y, et al. Estimates of the impact of extreme heat events on cooling energy demand in Hong Kong [J]. Renewable Energy, 2019, 142: 73-84.

[12] Santamouris M, Cartalis C, Synnefa A, et al. On the impact of urban heat island and global warming on the power demand and electricity consumption of buildings: A review [J]. Energy and Buildings, 2015, 98: 119-124.

[13] Sun Y, Augenbroe G. Urban heat island effect on energy application studies of office buildings [J]. Energy and Buildings, 2014, 77(7): 171-179.

[14] Arifwidodo S, Chandrasiri O. Urban heat island and household energy consumption in Bangkok, Thailand [J]. Energy Procedia, 2015, 79: 189-194.

[15] Ding F, Pang H J, Guo W H. Impact of the urban heat island on residents' energy consumption: A case study of Qingdao [J]. IOP Conference Series: Earth and Environmental Science, 2018, 121: 032026. doi: 10.1088/17551315/121/3/032026.

[16] Memon R A, Leung D Y C, Liu C H, et al. Urban heat island and its effect on the cooling and heating demands in urban and suburban areas of Hong Kong [J]. Theoretical and Applied Climatology, 2011, 103(3-4): 441-450.

[17] 黄勇波. 城市热岛效应对建筑能耗影响的研究 [D]. 天 津: 天津大学, 2005. [Huang Yongbo. Study on the impact of urban heat island on the energy consumption of 
buildings. Tianjin, China: Tianjin University, 2005. ]

[18] Meng F C, Li M C, Cao J F, et al. The effects of climate change on heating energy consumption of office buildings in different climate zones in China [J]. Theoretical and Applied Climatology, 2018, 133: 521-530.

[19] 田喆, 黄勇波, 朱能, 等. 城市热岛作用下办公建筑冷负 荷的影响因素分析 [J]. 流体机械, 2005, 33(5): 54-57. [Tian Zhe, Huang Yongbo, Zhu Neng, et al. Analysis for the impact of UHI on office building cooling load for different factors. Fluid Machinery, 2005, 33(5): 54-57. ]

[20] Li M C, Cao J F, Xiong M M, et al. Different responses of cooling energy consumption in office buildings to climatic change in major climate zones of China $[\mathrm{J}]$. Energy and Buildings, 2018, 173: 38-44.

[21] 陈莉, 方修睦, 方修琦, 等. 过去 20 年气候变暖对我国冬 季采暖气候条件与能源需求的影响 $[\mathrm{J}]$. 自然资源学 报, 2006, 21(4): 590-597. [Chen Li, Fang Xiumu, Fang Xiuqi, et al. Impacts of climate warming on heating climatic conditions and energy requirements over China in the past 20 years. Journal of Natural Resources, 2006, 21 (4): 590-597. ]

[22] 史军, 崔林丽, 田展, 等. 气温变化对华东居住建筑取暖 和降温耗能的影响 [J]. 自然资源学报, 2011, 26(3): 460-467. [Shi Jun, Cui Linli, Tian Zhan, et al. Impact of temperature change on heating and cooling energy consumption of residential buildings in East China. Journal of Natural Resources, 2011, 26(3): 460-467. ]

[23] Hwang R L, Lin C Y, Huang K T. Spatial and temporal analysis of urban heat island and global warming on residential thermal comfort and cooling energy in Taiwan [J]. Energy and Buildings, 2017, 152: 804-812.

[24] Cui Y, Yan D, Hong T Z, et al. Comparison of typical year and multiyear building simulations using a 55-year actual weather data set from China $[\mathrm{J}]$. Applied Energy, 2017, 195: 890-904.

[25] Ren Y Y, Ren G Y. A remote-sensing method of selecting reference stations for evaluating urbanization effect on surface air temperature trends $[\mathrm{J}]$. Journal of Climate, 2011, 24(13): 3179-3189.

[26] Yang P, Ren G Y, Liu W D. Spatial and temporal characteristics of Beijing urban heat island intensity [J]. Journal of Applied Meteorology and Climatology, 2013, 52(8): 1803-1816.

[27] 宁巍. 基于 TRNSYS 的变风量空调系统优化控制仿真 [D]. 哈尔滨: 哈尔滨工业大学, 2013. [Ning Wei. Simulation on the optimized control of VAV air- conditioning system based on TRNSYS. Harbin, China: Harbin Institute of Technology, 2013. ]

[28] 安册册. 数据中心空调系统预测模型研究 [D]. 北京: 北 京建筑大学, 2017. [An Cece. Research on forecast model of air conditioning system in data center. Beijing, China: Beijing University of Civil Engineering and Architecture, 2017. ]

[29] Magli S, Lodi C, Lombroso L, et al. Analysis of the urban heat island effects on building energy consumption [J]. International Journal of Energy and Environmental Engineering, 2015, 6(1): 91-99.

[30] Guattari C, Evangelisti L, Balaras C A. On the assessment of urban heat island phenomenon and its effects on building energy performance: A case study of Rome (Italy) [J]. Energy and Buildings, 2018, 158: 605-615.

[31] 李明财, 郭军, 史珺, 等. 气候变化对天津市商场和居住 建筑极端能耗的影响 [J]. 高原气象, 2014, 33(2): 574583. [Li Mingcai, Guo Jun, Shi Jun, et al. Effect of climate change on extreme energy consumption for residential and commercial buildings in Tianjin. Plateau Meteorology, 2014, 33(2): 574-583. ]

[32] Song X Y, Ye C T, Li H S, et al. Field study on energy economic assessment of office buildings envelope retrofi tting in southern China [J]. Sustainable Cities and Society, 2017, 28: 154-161.

[33] Li M C, Shi J, Guo J, et al. Climate impacts on extreme energy consumption of different types of buildings $[\mathrm{J}]$ PLoS One, 2015, 10(4): 1-12.

[34] 任芝花, 熊安元. 地面自动站观测资料三级质量控制业 务系统的研制 [J]. 气象, 2007, 33(1): 19-24. [Ren Zhihua, Xiong Anyuan. Operational system development on three-step quality control of observations from AWS. Meteorological Monthly, 2007, 33(1): 19-24. ]

[35] 李红莲, 吕凯琳, 杨柳. 气候变化下未来西安几种类型 建筑暖通空调负荷分析预测 [J]. 西安建筑科技大学学 报(自然科学版), 2018, 50(4): 549-555. [Li Honglian, Lv Kailin, Yang Liu. Analysis and prediction of the different building HVAC load under climate change for Xi'an Journal of Xi'an University of Architecture and Technology (Natural Science Edition), 2018, 50(4): 549-555. ]

[36] 高静, 龚健, 李靖业. “源一汇”景观格局的热岛效应研 究: 以武汉市为例 [J]. 地理科学进展, 2019, 38(11): 1770-1782. [Gao Jing, Gong Jian, Li Jingye. Effects of source and sink landscape pattern on land surface temperature: An urban heat island study in Wuhan City. Progress in Geography, 2019, 38(11): 1770-1782. ] 
[37] 谢正辉, 刘斌, 延晓冬, 等. 应对气候变化的城市规划实 施效应评估研究 [J]. 地理科学进展, 2020, 39(1): 120131. [Xie Zhenghui, Liu Bin, Yan Xiaodong, et al. Effects of implementation of urban planning in response to climate change. Progress in Geography, 2020, 39(1): 120131. ]

[38] Duan S P, Luo Z W, Yang X Y, et al. The impact of building operations on urban heat/cool islands under urban densification: A comparison between naturally-ventilated and air-conditioned buildings [J]. Applied energy, 2019, 235: $129-138$.

[39] 张雷, 任国玉, 任玉玉. 单次极端高温过程中城市热岛 效应的识别 [J]. 气候与环境研究, 2015, 20(2): 167176. [Zhang Lei, Ren Guoyu, Ren Yuyu. Identification of urban effect on a single extreme high temperature event. Climatic and Environmental Research, 2015, 20(2): 167176. ]

[40] 李明财, 熊明明, 任雨, 等. 未来气候变化对天津市居住 建筑制冷采暖能耗的影响 [J]. 气候变化研究进展, 2013, 9(6): 398-405. [Li Mingcai, Xiong Mingming, Ren $\mathrm{Yu}$, et al. Impact of climate change on office building energy consumption for cooling and heating in Tianjin, China. Advances in Climate Change Research, 2013, 9(6): 398-405. ]

[41] Santamouris M, Haddad S, Saliari M, et al. On the energy impact of urban heat island in Sydney: Climate and energy potential of mitigation technologies $[\mathrm{J}]$. Energy and Buildings, 2018, 166: 154-164.

[42] 陈怡. 广州城市热岛强度对建筑空调采暖能耗影响研
究 [D]. 广州: 华南理工大学, 2017. [Chen Y. Study on the impact of UHI intensity on the energy consumption of building air conditioning in Guangzhou. Guangzhou, China: South China University of Technology, 2017. ]

[43] 刘树华, 刘振金金, 李炬, 等. 京津冀地区大气局地环流耦 合效应的数值模拟 [J]. 中国科学: 地球科学, 2009, 39 (1): 88-98. [Liu Shuhua, Liu Zhenxin, Li Ju, et al. Numerical simulation of the coupling effect of local atmospheric circulation in Beijing-Tianjin-Hebei region. Scientia Sinica Terrae, 2009, 39(1): 88-98. ]

[43] 东高红, 尉英华, 解以扬, 等. 天津地区城市热岛环流与 海风环流相互作用的研究 [J]. 气象, 2015, 41(12): 1447-1455. [Dong Gaohong, Wei Yinghua, Xie Yiyang, et al. Research on the interaction of Tianjin urban heat island circulation and sea breeze circulation. Meteorological Monthly, 2015, 41(12): 1447-1455. ]

[45] Lee T W, Choi H S, Lee J. Generalized scaling of urban heat island effect and its applications for energy consumption and renewable energy [J]. Advances in Meteorology, 2014, 2014. doi: 10.1155/2014/948306.

[46] 刘玉莲, 任国玉. 基于度一时法的哈尔滨冬季采暖强度 评价 [J]. 自然资源学报, 2018, 33(1): 139-148. [Liu Yulian, Ren Guoyu. Analysis of heating intensity in Harbin based on degree- hours method. Journal of Natural Resources, 2018, 33(1): 139-148. ]

[47] Tewari M, Salamanca F, Martilli A, et al. Impacts of projected urban expansion and global warming on cooling energy demand over a semiarid region [J]. Atmospheric Science Letters, 2017, 18(11): 419-426. 


\title{
Impact of urban heat island effect on the heating and cooling loads of residential buildings in Tianjin City, China
}

\author{
MENG Fanchao', REN Guoyu ${ }^{2,3^{*}}$, GUO Jun', ZHANG Lei ${ }^{4}$, ZHANG Ruixue ${ }^{5}$ \\ (1. Tianjin Climate Center, Tianjin 300074, China; 2. Laboratory for Climate Studies, National Climate Center, \\ China Meteorological Administration, Beijing 100081, China; 3. Department of Atmospheric Science, \\ School of Environmental Studies, China University of Geosciences, Wuhan 430074, China; \\ 4. National Meteorological Information Center, China Meteorological Administration, Beijing 100081, China; \\ 5. China Academy of Building Research, Beijing 100013, China)
}

\begin{abstract}
The urban heat island (UHI) effect has had an important impact on building energy demand in big cities. It is of great significance, therefore, to evaluate the impact of the UHI on building energy demand in a metropolis in order to save energy for existing buildings and to better design for future buildings. Based on the hourly data from automatic weather stations and four representative rural weather stations selected by satellite remote-sensing method, the impacts of urban heat island intensity $\left(I_{\mathrm{UHI}}\right)$ on building heating and cooling loads of different time scales (annual, daily, and hourly) in Tianjing City were evaluated by simulating hour-by-hour loads of the typical residential buildings. The results show that: 1) The heating loads decreased and the cooling loads increased with the increase of the $I_{\mathrm{UнI}}$ in the past nine years. The decreased magnitude of the yearly heating load was larger than the increased magnitude of the yearly cooling load. When the $I_{\text {Uнг }}$ increased by $1{ }^{\circ} \mathrm{C}$, the annual mean heating loads decreased by $4.01 \mathrm{kWh} / \mathrm{m}^{2}$ and the annual mean cooling loads increased by 1.05 $\mathrm{kWh} / \mathrm{m}^{2}$ in the urban areas compared to the rural areas. 2) The heating loads were the highest in the period from late December to late January of the following year, and the daily mean loads in the urban areas were about $10 \%$ lower than that in the rural areas. The cooling loads were the highest from late July to early August, and the daily mean loads in urban areas were about $6 \%$ higher than that in the rural areas. 3) Both heating and cooling loads were higher during the nighttime than daytime. The period from 18:00 to 07:00 Beijing Time was the high load stage in the urban and rural areas, regardless of heating period or cooling period. From 11:00 to 15:00 Beijing Time, however, the loads were low in the heating period but high in the cooling period, which may have been caused by changing temperature and the different heating or cooling demand of the urban residents. Therefore, the impact of UHI on hourly and daily building loads should be fully considered to promote the effective management of urban heating and cooling operation for reducing building energy consumption in big cities of northern China like Tianjin.
\end{abstract}

Keywords: urban heat island effect; rural weather station; heating/cooling load; Tianjin City 